\title{
Expression, Purification and Transduction of PEP-1-Botulinum Neurotoxin Type A (PEP-1-BoNT/A) into Skin
}

\author{
Dae Won Kim ${ }^{\dagger}$, So Young Kim $\dagger$, Jae Jin An, Sun Hwa Lee, Sang Ho Jang, Moo Ho Won', \\ Tae-Cheon Kang ${ }^{1}$, Kwang-Hoe Chung', Hyun-Ho Jung ${ }^{3}$, Sung-Woo Cho ${ }^{4}$, \\ Jin Hi Choi ${ }^{5}$, Jinseu Park, Won Sik Eum and Soo Young Choi* \\ Department of Biomedical Sciences and Research Institute for Bioscience and Biotechnology, Hallym University \\ ${ }^{1}$ Department of Anatomy, College of Medicine, Hallym University, Chunchon 200-702, Korea \\ ${ }^{2}$ Biobud Co., Ltd, Yonhui-dong, Seoul 120-110, Korea \\ ${ }^{3}$ Medy-Tox Inc. Ochang Science Industrial Complex, Chungchungbuk-do 363-883, Korea \\ ${ }^{4}$ Department of Biochemistry and Molecular Biology, University of Ulsan College of Medicine, Seoul 138-736, Korea \\ ${ }^{5}$ Research Laboratory of Cell Tech. Korea, Chunchon 200-702, Korea
}

Received 14 May 2006, Accepted 5 June 2006

\begin{abstract}
Botulinum neurotoxin A (BoNT/A) has been used therapeutically to treat muscular hypercontractions and sudomotor hyperactivity and it has been reported that BoNT/A might have analgesic properties in headache. PEP-1 peptide is a known carrier peptide that delivers fulllength native proteins in vitro and in vivo. In this study, a BoNT/A gene were fused with PEP-1 peptide in a bacterial expression vector to produce a genetic in-frame PEP-1BoNT/A fusion protein. The expressed and purified PEP1-BoNT/A fusion proteins were efficiently transduced into cells in a time- and dose-dependent manner when added exogenously in a culture medium. In addition, immunohistochemical analysis revealed that PEP-1-BoNT/A fusion protein efficiently penetrated into the epidermis as well as the dermis of the subcutaneous layer, when sprayed on mice skin. These results suggest that PEP-1-BoNT/A fusion protein provide an efficient strategy for therapeutic delivery in various human diseases related to this protein.
\end{abstract}

Keywords: Botulinum toxin A (BoNT/A), Facial wrinkles, PEP-1 peptide, Protein therapy, Protein transduction

\footnotetext{
These authors contributed equally to this work

*To whom correspondence should be addressed. Tel: 82-33-248-2112; Fax: 82-33-241-1463

E-mail: sychoi@hallym.ac.kr
}

\section{Introduction}

Botulinum neurotoxins (BoNTs) are a group of homologous protein toxins, produced by various strains of Clostridium botulinum and in some cases Clostridium butyricum and Clostridium baratii (Schiavo et al., 2000). Seven immunologically distinct forms of the neurotoxin, BoNT (A-G), have been identified and released by the clostridia as inactive 150 $\mathrm{kDa}$ protein precursors (Oguma et al., 1995; Lacy et al., 1999). These proteins are the most toxic substances known and are the cause of flaccid paralysis in botulism. Naturally occurring botulism is found in infants or adults gastrointestinal tracts become colonized by Clostidial bacteria (infant or intestinal botulinum), after ingestion of contaminated food products (food botulinum), or in anaerobic wound infections (wound botulinum) (Centers for Disease Control, 1998). BoNTs are the most potent natural toxins known humankind with a mouse $50 \%$ lethal dose range of $0.1-1 \mathrm{ng} / \mathrm{kg}$ of body weight (Schiavo et al., 2000). BoNT acts on the peripheral nervous system by inhibiting the release of acetylcoline at neuromuscular junctions resulting in progressive flaccid paralysis (Sugiyama, 1980).

BoNT/A is produced as single inactive polypeptide chains of $150 \mathrm{kDa}$, which subsequently undergo endogenously or exogenously proteolytic cleavage to yield the fully active dichain molecule comprising of a $100 \mathrm{kDa}$ heavy chain and a 50 $\mathrm{kDa}$ light chain linked via both non-covalent interactions and a disulfide bond, the integrity of which is essential for the neurotoxicity. The heavy chain is further divided into two domains, each with a specific function. The C-terminal half of the heavy chain binds to the cell surface. The N-terminal domain is thought to form "channels" in the endosomal 
membrane and to facilitate the transport of the light chain through the endosomal membrane into the cytoplasm. The light chain is the zinc-dependent catalytic domain of the toxin that targets and cleaves one of three SNARE proteins VAMP/ synaptobrevin, syntaxin, and SNAP-25 that are essential for synaptic vesicle fusion. Cleavage of the SNARE proteins results in inhibition of acetylcholine secretion, which leads to paralysis (Montecucco and Schiavo, 1994; Schmidt and Bostian, 1995).

The BoNTs are best known for their role in mediating foodborne, wound and infant botulism. Their high potency and ease of production also render the BoNTs potential biological warfare agents (Christopher et al., 1997; Arnon et al., 2001). Recently, BoNTs are increasingly being used as therapeutic agents to treat several neurological disorders as well as for cosmetic purposes to remove facial wrinkles and frown lines. The efficacy of BoNTs in the treatment of pain syndromes, including migraine and myofiscal pain, has been well demonstrated (Wheeler, 1997; Cordivari et al., 2004; Klein, 2004).

Up to the present, many researchers have demonstrated the successful delivery of full-length Tat fusion proteins by protein transduction technology (Wadia and Dowdy, 2002). Recently, several small regions of proteins, called protein transduction domains (PTDs), have been developed to allow the delivery of exogenous protein into living cells (Fawell et al., 1991; Vives et al., 1997; Prochiantz, 2000; Morris et al., 2002). We also successfully transduced Tat-SOD directly into various cell lines including pancreatic islet cells and found that transduced Tat-SOD increased radical scavenger activity (Eum et al., 2004a). More recently, we transduced PEP-1SOD and Tat-SOD into neuronal cells across the blood-brain barrier and found that it efficiently protected against ischemic insults and paraquat-induced neurodegeneration (Eum et al., 2004b; Kim et al., 2005; Choi et al., 2006).

In this study, we designed the PEP-1-BoNT/A fusion protein for direct transduction in vitro and in vivo. The successes of PEP-1-BoNT/A fusion protein transduction may suggest that this fusion protein would be useful as cosmetic for treatment of facial wrinkles and therapeutic agents.

\section{Materials and Methods}

Construction, expression and purification of PEP-1-BoNT/A. The PEP-1-SOD expression vector was constructed as previously described (Eum et al., 2004b) and the PEP-1-BoNT/A was constructed as following procedures. Briefly, synthesized oligonucleotide was ligated into NdeI-XhoI-digested pET-15b vector. Next, on the basis of the light chain cDNA sequence of BoNT/A, two primers were synthesized. The sense primer, 5'-CTCGAGATGCCCTTTGT TAATAAACAATTTAAT-3' contains an XhoI site, and the antisense primer, 5'-GGATCCTGACTTATTGTATCCTTCATCTAATGA-3', contains a BamHI restriction site. The PCR reaction was performed and the PCR product was excised with $X h o \mathrm{I}$ and BamHI, eluted (Invitek), ligated into TA-cloning vector (Promega) and pPEP-1 vector using T4 DNA ligase (Takaka), and cloned in E. coli DH5a cells. PEP-1-BoNT/A fusion proteins were generated when the BoNT/A gene was fused with a 21 amino acid PEP-1-peptide in a bacterial expression vector in order to produce a genetic in-frame PEP-1-BoNT/A fusion protein.

To produce the PEP-1-BoNT/A fusion protein, the plasmid was transformed into $E$. coli $\mathrm{BL} 21$ cells. The transformed bacterial cells were grown in $100 \mathrm{ml}$ of $\mathrm{LB}$ media at $37^{\circ} \mathrm{C}$ to a $D_{600}$ value of $0.5 \sim 1.0$ and induced with $0.5 \mathrm{mM}$ IPTG at $30^{\circ} \mathrm{C}$ for $12 \mathrm{~h}$. Harvested cells were lysed by sonication at $4^{\circ} \mathrm{C}$ in a binding buffer $(5 \mathrm{mM}$ imidazole, $500 \mathrm{mM} \mathrm{NaCl}, 20 \mathrm{mM}$ Tris- $\mathrm{HCl}, \mathrm{pH} 7.9$ ), and the recombinant PEP-1-BoNT/A formed was purified. Briefly, clarified cell extracts were loaded onto a $\mathrm{Ni}^{2+}$-nitrilotriacetic acid Sepharose affinity column (Qiagen) under native conditions. After the column was washed with 10 volumes of a binding buffer and six volumes of a wash buffer ( $35 \mathrm{mM}$ imidazole, $500 \mathrm{mM} \mathrm{NaCl}$, and $20 \mathrm{mM}$ Tris-HCl, $\mathrm{pH}$ 7.9), the fusion proteins were eluted using an eluting buffer (0.5 M imidazole, $500 \mathrm{mM} \mathrm{NaCl}, 20 \mathrm{mM}$ Tris-HCl, $\mathrm{pH}$ 7.9). The fusion protein containing fractions was combined and the salts were removed using a PD-10 column (Amersham). The protein concentration was estimated by the Bradford procedure using bovine serum albumin as a standard (Bradford, 1976).

Transduction of PEP-1-BoNT/A into HeLa cells. For the transduction of PEP-1- BoNT/A, HeLa cells were grown to confluence on a 6 well plate. And then the culture medium was replaced with $1 \mathrm{ml}$ of fresh solution. After the cells were treated with various concentrations of PEP-1-BoNT/A for $1 \mathrm{~h}$, the cells were treated with trypsin-EDTA (Gibco) and washed with phosphate-buffered saline (PBS). The cells were harvested for the preparation of cell extracts to perform a Western blot analysis (Kim, 2003; Ha et al., 2004).

The intracellular stability of transduced PEP-1-BoNT/A fusion protein was estimated as follow: After the cells were treated with $3 \mu \mathrm{M}$ PEP-1-BoNT/A for $1 \mathrm{~h}$, the cells were washed and changed with a fresh culture medium to remove PEP-1-BoNT/A that was not transduced. Cells were further incubated for $72 \mathrm{~h}$ and extracted for Western blot analysis.

Fluorescence analysis. For direct detection of fluorescein-labeled protein, purified PEP-1-BoNT/A was labeled using EZ-Label fluorescein isothiocyanate (FITC) protein labeling kit (PIERCE). The labeling of FITC was carried out according to the manufacturer's instruction. Cultured cells were grown on glass coverslips and treated with $3 \mu \mathrm{M}$ PEP-1-BoNT/A fusion proteins. Following incubation for $1 \mathrm{~h}$ at $37^{\circ} \mathrm{C}$, the cells were washed twice with PBS and trypsin-EDTA. After fixing the cells with $4 \%$ paraformaldehyde for $10 \mathrm{~min}$ at room temperature, the distribution of fluorescence was analyzed on a fluorescence microscopy (Carl Zeiss) as described elsewhere (Hwang et al., 2004).

Immunohistochemistry. Mice were housed at a constant temperature $\left(23^{\circ} \mathrm{C}\right)$ and relative humidity $(60 \%)$ with a fixed $12 \mathrm{~h}$ light/dark cycle and free access to food and water. Procedures involving animals and their care conformed to the institutional guidelines, which are in compliance with current international laws and policies (NIH Guide for the Care and Use of Laboratory Animals, NIH Publication No. 85-23, 1985, revised 1996) and were 
approved by the Hallym Medical Center Institutional Animal Care and Use Committee. While the animals were anesthetized with 3\% isoflurance in nitrogen and oxygen, $50 \mathrm{mg}$ control and PEP-1BoNT/A fusion proteins were topically applied onto the shaved area of the mice skin for $1 \mathrm{~h}$, respectively. Thereafter, frozen and sectioned tissues were prepared and fixed with $4 \%$ paraformaldehyde for $10 \mathrm{~min}$. For removal of non-specific immunoreactivity, freefloating sections were first incubated with $0.3 \%$ Triton X-100 and $10 \%$ normal goat serum in PBS for $1 \mathrm{~h}$ at room temperature. They were then incubated with a rabbit anti-histidine $\operatorname{IgG}(1: 500)$ for $24 \mathrm{~h}$ at room temperature. After washing three times for $10 \mathrm{~min}$ with PBS, the sections were incubated for $1 \mathrm{~h}$ with biotinylated goat anti-rabbit IgG (Vector Laboratories, USA; dilution $1: 200$ ), then visualized with 3,3'-diaminobenzidine (40 mg DAB/0.045\% $\mathrm{H}_{2} \mathrm{O}_{2}$ in $100 \mathrm{ml}$ PBS) mounted on gelatin-coated slides. The immunoreactions were observed under an Axioscope microscope (Carl Zeiss).

\section{Results and Discussion}

Botulinum toxin A (BoNT/A) has a wide variety of clinical applications, which are related by blockade of acetylcholine and often are related to abnormal muscle contractures. These applications include several neurological disorders, migraine, headache, and cosmetics (Mauskop and Basdeo, 2000; Verheyden and Blitzer, 2002; Mauskop, 2002; Klein, 2004).

Wrinkles are known to be a consequence of the natural aging process. They results from three factors; age influencing by photoaging, gravity favoring the breakdown of elastic fibers, and the action of the muscles of expression accentuating all kinds of wrinkles. There are two types of wrinkles due to aging and hyperkinesis (Sarrabayrouse, 2002). Wrinkles can be defined by their morphological structure and their depth, which can be superficial, intermediated or deep. Mid peels are suitable for superficial wrinkles, whereas fillers and deep peels are suitable for intermediate and deep wrinkles. Associated wrinkles present static and dynamic components. It is usually better to treat the dynamic component of the associated wrinkle first, and its static component afterwards (De Maio, 2003). Also, BoNT/A is an import agent in facial rejuvenation and it is an adjunctive procedure to surgical treatment (Carruthers and Carruthers, 1998).

In this work, we are describing a genetic approach of the PEP-1-BoNT/A fusion protein into the HeLa cells and animal skin. To develop an expression system to overexpress and purify simply the cell-permeable BoNT/A protein, we constructed the PEP-1-BoNT/A expression vector, which contains consecutive cDNA sequences encoding BoNT/A, PEP-1 peptide (21 amino acids), and six histidine residues at the amino terminus (Fig. 1). Also, we constructed the BoNT/ A expression vector to produce control BoNT/A protein without PEP-1 transduction peptides (data not shown).

Following the induction of expression, PEP-1-BoNT/A and BoNT/A fusion proteins was purified using $\mathrm{Ni}^{2+}$-nitrilotriacetic acid Sepharose affinity column and PD-10 column. SDS-
(A)

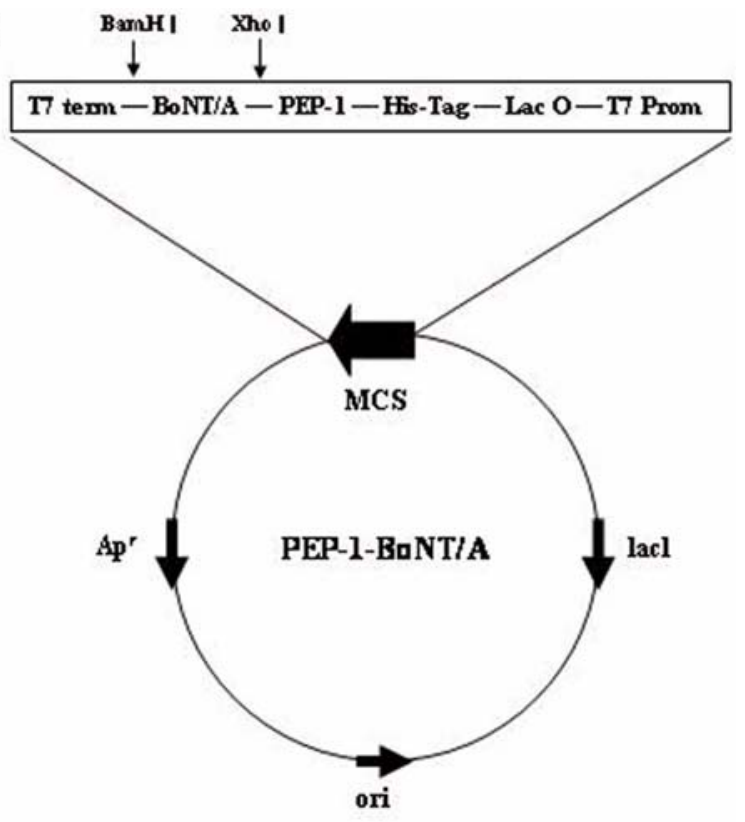

(B)

PEP-1-BoNT/A

His-Tag - PEP-1 -BoNT/A

Control BoNT/A

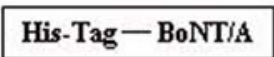

Fig. 1. The expression vector for PEP-1-BoNT/A fusion protein. (A) Construction of the PEP-1-BoNT/A expression vector system based on the vector pET-15b. Synthetic PEP-1 oligomer was cloned with into the NdeI, XhoI sites, and human BoNT/A cDNA was cloned into the XhoI, BamHI sites of pET-15b. (B) Diagram of expressed control BoNT/A and PEP-1-BoNT/A fusion proteins. Expression was induced by adding $0.5 \mathrm{mM}$ IPTG.

PAGE and Western blot analysis of the purified PEP-1-BoNT/ A was performed. As shown in Fig. 2A, PEP-1-BoNT/A was highly expressed and was a major component of the total soluble proteins. The recombinant PEP-1-BoNT/A protein had estimated molecular masses of approximately $53 \mathrm{kDa}$. However, it was detected that recombinant fusion protein migrated to bands with a higher molecular weight than those of the expected sizes on the SDS-PAGE. The purified proteins were confirmed by Western blot analysis using an anti-rabbit polyhistidine antibody. PEP-1-BoNT/A was detected at the corresponding bands in Fig. 2B.

The Morris group (2001) designed a 21-residue peptide carrier, PEP-1 (KETWWETWWTEWSQPKKKRKV), which consists of three domains: a hydrophobic tryptophan rich motif (KETWWETWWTEW), a spacer (SQP), and a hydrophilic lysine-rich domain (KKKRKV). The hydrophobic domain is required for efficiently targeting the cell membrane and for hydrophobic interactions with proteins, whereas the hydrophilic domain is required to improve intracellular delivery. When mixed PEP-1 peptide and target protein (GFP, $\beta$-gal) were overlaid on cultured cells, it was found that the non-denatured target protein was transduced. Recently, we reported that PEP- 
(A)

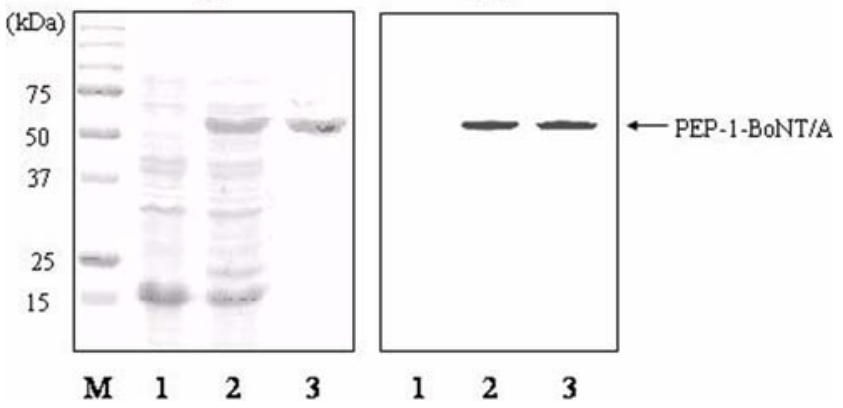

Fig. 2. Expression and purification of PEP-1-BoNT/A fusion protein. Expressed and purified fusion proteins were analyzed by $12 \%$ SDS-PAGE (A) and subjected to Western blot analysis with an anti-rabbit polyhistidine antibody (B). Lanes are as follow: lane 1, non-induced; lane 2, induced; lane 3, purified PEP-1-BoNT/A.
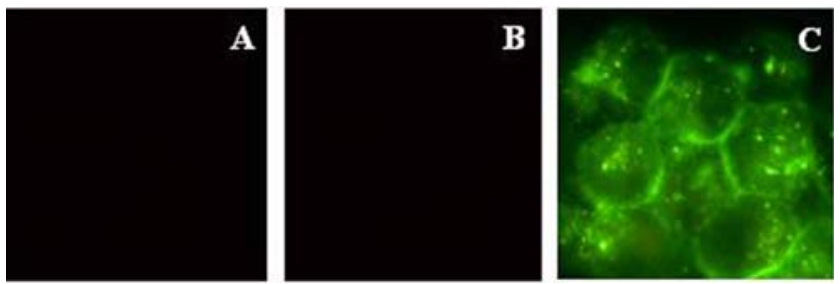

Fig. 3. Cellular localization of PEP-1-BoNT/A. After FITClabeled PEP-1-BoNT/A $(3 \mu \mathrm{M})$ was transduced into HeLa cells, the cells were washed twice with trypsin-EDTA followed by PBS and immediately observed by fluorescence microscopy. Control cell itself without PEP-1-BoNT/A (A), cells treated with control BoNT/A (B), and cells treated with PEP-1-BoNT/A (C).

1-SOD fusion protein is transduced in vitro and in vivo and protects the neuronal cells from destruction by reactive oxygen species (ROS) (Eum et al., 2004b).

The intracellular delivery of PEP-1-BoNT/A into HeLa cells was confirmed by direct fluorescence used FITC conjugated PEP-1-BoNT/A fusion protein. As shown in Fig. 3C, almost all cultured cells were found to be transduced with PEP-1BoNT/A, whereas fluorescence signals were not detected in cells treated with control BoNT/A (Fig. 3A and 3B). In addition, we confirmed that cell fixation with paraformaldehyde is not required for PEP-1-BoNT/A fusion protein transduction (data not shown).

To evaluate the transduction ability of PEP-1-BoNT/A fusion protein, we analyzed the transduction of PEP-1-BoNT/ A proteins by adding them to HeLa cell culture medium at $3 \mathrm{mM}$ for various periods of time $(10-60 \mathrm{~min})$, and then analyzed the transduced protein levels by Western blotting. The intracellular concentration of transduced PEP-1-BoNT/A in cells was detected within $10 \mathrm{~min}$ and gradually increased up to $60 \mathrm{~min}$ (Fig. 4A). The dose-dependency of the transduction of PEP-1-BoNT/A fusion proteins was further analyzed. Various concentrations (0.5-3 mM) of PEP-1-BoNT/A proteins
(A)

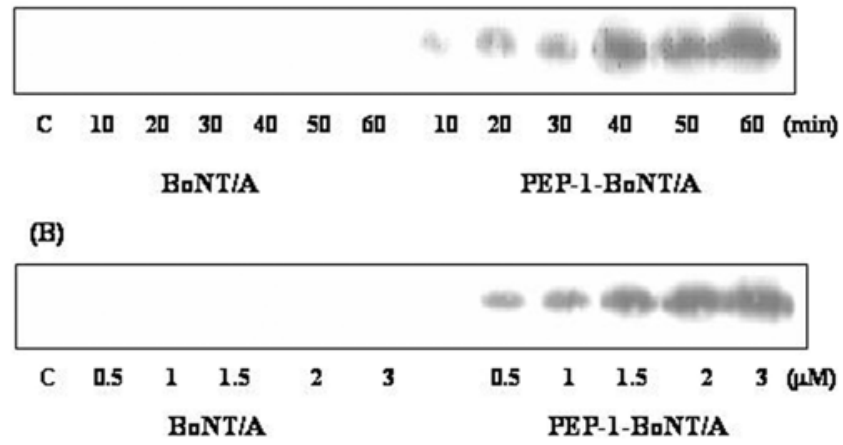

Fig. 4. Transduction of $\mathrm{PEP}-1-\mathrm{BoNT} / \mathrm{A}$ fusion protein into cultured HeLa cells. (A) $3 \mu \mathrm{M}$ of PEP-1-BoNT/A and control $\mathrm{BoNT} / \mathrm{A}$ were added to the cultured media for various time, respectively. (B) $0.5-3 \mu \mathrm{M}$ of PEP-1-BoNT/A and control BoNT/A were added to the cultured media for $60 \mathrm{~min}$, respectively. Transduced PEP-1-BoNT/A into the cells was analyzed by Western blotting.

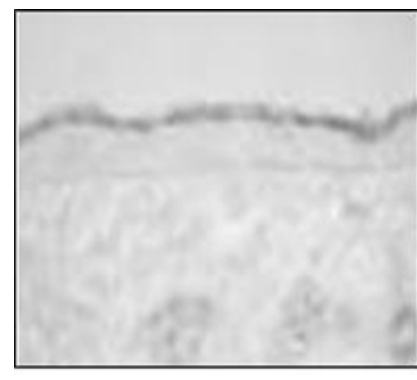

Contral

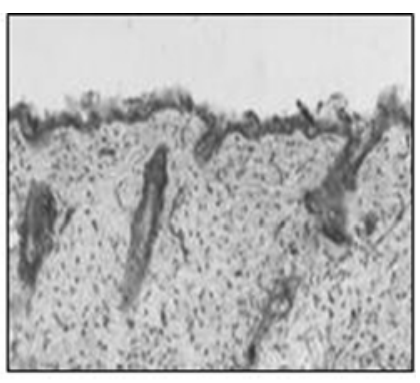

PEP-1-B口NT/A
Fig. 5. Histochemical analysis of animal skin transduced with PEP-1- BoNT/A fusion protein. PEP-1-BoNT/A fusion proteins $(50 \mu \mathrm{g})$ were topically applied onto the shaved area of mouse dorsal skin for $60 \mathrm{~min}$. Frozen sections of skin tissues were immunostained with rabbit anti-histidine $\operatorname{IgG}(1: 400)$ and then stained with biotinylated goat anti-rabbit $\operatorname{IgG}(1: 200)$. The sections were visualized with 3,3'-diaminobenzidine and observed under an Axioscope microscope.

were added to HeLa in culture for $60 \mathrm{~min}$, and the levels of transduced proteins were measured by Western blotting. As shown in Fig. 4B, the higher level of transduction into the cells was observed as increasing the amount of fusion protein in the culture medium. Next, the intracellular stability of transduced PEP-1-BoNT/A into HeLa cells was examined by Western blotting. After the cells were treated with $3 \mathrm{mM}$ PEP1-BoNT/A for $1 \mathrm{~h}$, the cells was washed, changed with a fresh culture medium, and further incubated up to $72 \mathrm{~h}$. The intracellular level of transduced PEP-1-BoNT/A into cells was initially detected after $1 \mathrm{~h}$, and then declined gradually over the period of observation. However, significant levels of transduced PEP-1-BoNT/A persisted in the cells up to $48 \mathrm{~h}$ (data not shown).

A recent study showed that PEP-1 peptide/GFP (green 
fluorescent protein, $30 \mathrm{kDa}$ ) or $\beta$-Gal (b-galactosidase, 119 $\mathrm{kDa}$ ) mixtures transduce into a human fibroblast cell line (HS68) and into COS-7 cells by incubating with PEP-1 peptide carrier and proteins (GFP or $\beta$-gal) for $30 \mathrm{~min}$ at $37^{\circ} \mathrm{C}$ (Morris et al., 2001). In addition, we reported that PEP-1-SOD and pyridoxine-5-P Oxidase tranduced into astrocyte cells and PC12 cells for $30 \mathrm{~min}$, respectively (Eum et al., 2004b; Kim et al., 2006) and the PEP-1-CCS, copper chaperone for SOD fusion protein, took $60 \mathrm{~min}$ for transduction into astrocyte (Choi et al., 2005). Thus, these differences in the time courses of transduction may depend on whether the target protein is fused with the PEP-1 peptide. In case of the fusion proteins with the PEP-1 peptide, it may be possible that the conformation, the polarity, and the molecular shape of target proteins were altered after fusion with PEP-1 peptide and subsequently the transduction efficiency of fusion proteins into cells was improved.

We then examined the ability of PEP-1-BoNT/A fusion protein to transduce into mouse skin. The fusion protein proteins were sprayed on mice skin for $1 \mathrm{~h}$ and the degree of penetration of these fusion proteins was analyzed by immunohistochemistry. As shown in Fig. 5, transduction signals were clearly detected in the epidermis and dermis of skins treated with PEP-1-BoNT/A fusion proteins. Unlike PEP-1-BoNT/A, control BoNT/A could not transduce into skin. These results demonstrate that PEP-1-BoNT/A fusion protein can not only transduce into cultured cells but also penetrate the living animal cells.

PEP-1 peptide carrier presents several advantages for protein therapy, which include the translocation of native protein, high stability, a lack of toxicity, and a lack of sensitivity to serum. In particular, no toxicity to PEP-1 peptide was observed in several cell lines at up to $0.1-1.0 \mathrm{mM}$, while cell viability was only decreased by about $10 \%$ for PEP- 1 concentration of 1.0 $\mathrm{mM}$. Although the exact mechanisms of protein transduction are unclear, transduction of the BoNT/A fused with PEP-1 vector offers more attractive advantages for in vitro and in vivo protein therapy.

In summary, we demonstrate that BoNT/A fused with PEP1 peptide (PEP-1-BoNT/A) can efficiently transduce into cultured cells and penetrate into animal skin. Our success in the protein transduction of PEP-1-BoNT/A may provide a new strategy for protein therapy of various disorders related to this protein and for applications of ointment instead of hypodermic injection to remove facial wrinkles.

Acknowledgments This work was supported by a Next Generation Growth Engine Program Grant and the $21^{\text {st }}$ Century Brain Frontier Research Grant (M103KV01002006K2201-02010) from Korean Science and Engineering Foundation and in part by Regional Innovation Center (RIC) Grant from the Korean Ministry of Commerce, Industry and Energy.

\section{References}

Arnon, S. A., Schecter, R., Inglesby, T. V., Henderson, D. A., Bartlett, J. G., Ascher, M. S., Eitzen, E., Fine. A. D., Hauer, J., Layton, M., Lillibridge, S., Osterholm, M. T., O'Toole, T., Parker, G., Perl, T. M., Russell, P. K., Swerdlow, D. L. and Tonat, K. (2001) Botulinum toxin as a biological weapon: medical and public health management. J. Am. Med. Assoc. 285, 1059-1070.

Bradford, M. (1976) A rapid and sensitive method for the quantitation of microgram quantities utilizing the principle of protein-dye binding. Anal. Biochem. 72, 248-254.

Carruthers, J. and Carruthers, A. (1998) The adjunctive usage of botulinum toxin. Dermatol. Surg. 24, 1244-1247.

Center for Disease Control (1998) Botulism in the United States, 1899-1998. Handbook for Epidemiologists, Clinnicans, and Laboratory Workers. Atlanta, Georgia US Department of Health and Human Services, Public Health Service.

Choi, H. S., An, J. J., Kim, S. Y., Lee, S. H., Kim, D. W., Yoo, K. Y., Won, M. H., Kang, T. C., Kwon, H. J., Kang, J. H., Cho, S. W., Kwon, O. S., Park, J., Eum, W. S. and Choi, S. Y. (2006) PEP-1-SOD fusion protein efficiently protects against paraquat-induced dopaminergic neuron in Parkinson's Disease mouse model. Free Radic. Biol. Med. In press.

Choi, S. H., Kim, D. W., Kim, S. Y., An, J. J., Lee, S. H., Sohn, E. J., Hwang, S. I., Won, M. H., Kang, T. C., Kwon, H. J., Kang, J. H., Cho, S. W., Park, J., Eum, W. S. and Choi, S. Y. (2005) Transduced human copper chaperone for $\mathrm{Cu}, \mathrm{Zn}-\mathrm{SOD}$ (PEP-1-CCS) protects against neuronal cell death. Mol. Cells 20, 401-408.

Christopher, G. W., Cieslak, T. J., Pavlin, J. A. and Eitzen Jr, E. M. (1997) Biological warfare. A historical perspective. J. Am. Med. Assoc. 278, 412-417.

Cordivari, C., Misra, P. V., Catania, S. and Lees, A. J. (2004) New therapeutic indications for botulinum toxins. Mov. Disord. 19, 157-161.

De Maio, M. (2003) Botulinum toxin in associated with other rejuvenation methods. J. Cosmetic Laser Ther. 5, 210-212.

Eum W. S., Choung, I. S., Li, M. Z., Kang, J. H., Kim, D. W., Park, J., Kwon, H. Y. and Choi, S. Y. (2004a) HIV-1 Tat mediated protein transduction of $\mathrm{Cu}, \mathrm{Zn}$-superoxide dismutase into pancreatic b cells in vitro and in vivo. Free Radic. Biol. Med. 37, 339-349.

Eum, W. S., Kim, D. W., Hwang, I. K., Yoo, K. Y., Kang, T. C., Jang, S. H., Choi, H. S., Choi, S. H., Kim, Y. H., Kim, S. Y., Kwon, H. Y., Kang, J. H., Kwon, O. S., Cho, S. W., Lee, K. S., Park, J., Won, M. H. and Choi, S. Y. (2004b) In vivo protein transduction: Biologically active intact PEP-1superoxide dismutase fusion protein efficiently protects against ischemic insult. Free Radic. Biol. Med. 37, 1656-1669.

Fawell, S., Seery, J. and Daikh, Y. (1991) Tat-mediated delivery of heterologous proteins into cells. Proc. Natl. Acad. Sci. USA 91, 664-668.

Ha, K. T., Lee, Y. C., Cho, S. H., Kim, J. K. and Kim, C. H. (2004) Molecular characterization of membrane type and ganglioside-specific sialidase (Neu3) expressed in E. coli. Mol. Cells 17, 267-273. 
Hwang, J. H., Yuk, S. H., Lee, J. H., Lyoo, W. S., Ghil, S. H., Lee, S. S., Khang, I. G., Paik, S. Y. and Lee, J. Y. (2004) Differentiation of stem cells isolated from rat smooth muscle. Mol. Cells 17, 57-61.

Kim, C. H. (2003) A Salmonella typhimurium rfaE mutants recovers invasiveness for human epithelial cells when complemented by wild type $r f a E$ (confering biosynthesis of ADP-L-glycero-Dmanno-heptose-containing lipopolysaccharide). Mol. Cells 15, 226-232.

Kim, D. W., Eum, W. S., Jang, S. H., Kim, S. Y., Choi, H. S., Choi, S. H., An, J. J., Lee, S. H., Lee, K. S., Han, K., Kang, T. C., Won, M. H., Kang, J. H., Kwon, O. S., Cho, S. W., Kim, T. Y., Park, J. and Choi, S. Y. (2005) Transduced TatSOD fusion protein protects against ischemic brain injury. Mol. Cells 19, 88-96.

Kim, S. Y., An, J. J., Kim, D. W., Choi, S. H., Lee, S. H., Hwang, S. I., Kwon, O. S., Kang, T. C., Won, M. H., Cho, S. W., Park, J., Eum, W. S., Lee, K. S. and Choi, S. Y. (2006) Tat-mediated Protein Transduction of Human Brain Pyridoxine5-P Oxidase into PC12 Cells. J. Biochem. Mol. Biol. 39, 76-83.

Klein, A. W. (2004) The therapeutic potential of botulinum toxin. Dermatol. Surg. 30, 452-455.

Lacy, B. D. and Stevens, R. C. (1999) Sequence homology and structural analysis of the clostridial neurotoxins. J. Mol. Biol. 291, 1091-1104.

Mauskop, A. (2002) The use of botulinum toxin in the treatment of headaches. Curr. Pain Headache Rep. 6, 320-323.

Mauskop, A. and Basdeo, R. (2000) Botulinum toxin A is an effective prophylactic therapy for migraines. Cephalagia 20, 422.
Montecucco, C. and Schiavo, G. (1994) Mechanism of action of tetanus and botulinum neurotoxins. Mol. Microbiol. 13, 1-8.

Morris, M. C., Depollier, J., Mery, J., Heitz, F. and Divita, G. (2001) A peptide carrier for the delivery of biologically active proteins into mammalian cells. Nat. Biotechnol. 19, 1173-1176.

Oguma, K., Fujinaga, Y. and Inoue, K. (1995) Structure and function of Clostridium botulinum toxins. Microbiol. Immunol. 39, 161-168.

Prochiantz, A. (2000) Messenger proteins: homeoproteins, TAT and others. Curr. Opin. Cell Biol. 12, 400-406.

Sarrabayrouse, M. A. M. (2002) Indications and limitations for the use of botulinum toxin for the treatment of facial wrinkles. Aesth. Plast. Surg. 26, 233-238.

Schiavo, G., Matteoli, M. and Montecucco, C. (2000) Neurotoxins affecting neuroexocytosis. Physiol. Rev. 80, 717-766.

Schmidt, J. J. and Bostian, K. A. (1995) Proteolysis of synthetic peptides by type A botulinum neurotoxin. J. Protein Chem. 14, 703-708.

Sugiyama, H. (1980) Clostidium botulinum neurotoxin. Microbiol. Rev. 44, 419-448.

Verheyden, J. and Blitzer, A. (2002) Other noncosmetic uses of BOTOX. Dis. Mon. 48, 357-366.

Vives, E., Brodin, P. and Lebleu, B. (1997) A truncated HIV-1 Tat protein basic domain rapidly translocates through the plasma membrane and accumulates in the cell nucleus. J. Biol. Chem. 272, 16010-16017.

Wadia, J. S. and Dowdy, S. F. (2002) Protein transduction technology. Curr. Opin. Biotechnol. 13, 52-56.

Wheeler, A. H. (1997) Therapeutic uses of botulinum toxin. Am. Fam. Physican 55, 541-545, 548. 